\title{
Summaries of papers read at the meeting of the Society for Social Medicine 16 to 18 September 1976
}

Perinatal mortality in home and hospital booked cases Newcastle upon Tyne, 19601969. S. L. BARRON, A. M. THOMSON, AND P. R. PHILIPS

During the decade 1960-69, 33871 mothers in Newcastle upon Tyne were booked for delivery either in specialist hospitals or at home. Confinements in hospital rose from $45 \%$ in $1960-62$ to $80 \%$ in $1966-69$ and at the same time perinatal mortality fell from 36 to 26 per 1000 births. While home bookings fell from $51 \%$ to $23 \%$, the proportion of women so booked who were eventually transferred to hospital, rose from $13 \%$ to $21 \%$.

Perinatal mortality from 'obstetrical' causes fell at a greater rate than those from 'environmental' causes. Cases transferred from home to hospital showed a high mortality, which fell steeply from 128 (1960-62) to 54 (1966-69) per 1000 births. There is evidence of improved selection both at booking and at transfer, which probably accounts for the fall in the mortality rate among home deliveries. The above findings cannot be explained by changes in the distribution of social class, parity, age, maternal height, or birth weight nor by selective migration from the city.

Social processes in the development of disability and handicap. MICHAEL R. BURY

Traditional views of chronic sickness and disability have tended to stress either the individual or whole populations. The contribution of a sociological model should aim to integrate subjective responses to chronic sickness and the contexts in which they occur. The reported study is based on home interviews and observations at outpatient consultations of 25 individuals with suspected rheumatoid arthritis. From both patient and professional viewpoints there are difficulties in recognising illness. The individual is influenced by knowledge derived from personal experience, and by the more general cultural appreciation of arthritis. Help-seeking behaviour relates to perceived outcomes of contact with services and the effect such action will have on social life. Specialties such as rheumat- ology, and medical services in general, are critically evaluated by patients who have detailed experience of chronic sickness in daily settings. This study indicates that the social realities of chronic sickness and disability could play a much greater role in the shaping of policy and the organisation of services than they have done in the past.

Urban deprivation and health indicators. VERA CARSTAIRS

Data for Glasgow and Edinburgh Wards (37 and 23) were analysed. All wards were scored on a first principal component derived from census data indicating deprivation. These scores clearly reflected the known social pathology of these two cities, although analysis at ward level obscures the extremes of the range. Health indicators were in respect of mortality, hospital admissions, and birthweight; these data were standardised for age and sex to eliminate the effect of population structure, and data were aggregated over two to four years to give larger numbers of events. Correlations were calculated between the urban deprivation scores and the values of the individual health indicators. Some remarkably high correlations were observed-for example,

$\begin{array}{crc}\begin{array}{l}\text { Deaths } \\ \text { respiratory }\end{array} & \text { Glasgow } & \text { Edinburgh } \\ \text { disorders } & 0.883 & 0.683 \\ \text { perinatal } & 0.591 & \\ \text { General hospital admissions } & \\ \text { poisoning } & 0.645 & 0.794 \\ \text { all causes } & 0.579 & 0.472\end{array}$

The data provide a basis for health service policy although they do not indicate whether health services will provide an effective agent of intervention.

Evaluation of a geriatric screening scheme based on a health visitor questionnaire. M. J. RIGBY AND G. K. HEATON

A structured questionnaire was administered by health visitors to 500 elderly persons who had not recently presented to their general practitioner. In order to evaluate the effectiveness of the questionnaire in identifying serious unknown treatable morbidity, the subjects were then offered an examination by their genery practitioner, and the results compared. In the representative sample of elderly persors involved, the study identified 117 items of serious morbidity; 35 of these were nof previously known to the general prae titioner and were treatable, representing prevalence of $6.6 \%$ of persons examined The diagnostic questionnaire used alone as a screening method would have detected $88 \%$ of these latter conditions. The cost of the theoretical screening scheme wa calculated at 1975 rates as $£ 1.52$ per participant, or $£ 26$ per case of detected but previously unknown serious treatab寃 morbidity.

Peripheral outpatient clinics and outpapesif workload in Oxfordshire. M. J. GOLD貸E Outpatient bookings for the main medica and surgical specialities were surveye all hospitals and clinics in the Oxfordshie area during a four-week period (Jung 1975). 1853 of 20085 patients $(9 \%)$ were booked to peripheral clinics (that is, those

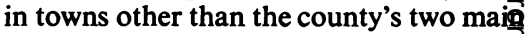
medical centres at Oxford and Banbury?. $53 \%$ of patients booked to periphera clinics lived in the same town as the clinic, and $75 \%$ lived either in the same town of in parishes adjacent to it. The age and ses distribution of patients seen at peripher and central clinics was similar and, in particular, locally held clinics did not take a greater proportion either of children of of the elderly than central clinics. A higher proportion of patients kept their appoin? ments at peripheral clinics (92\%) than central clinics $(87 \%)$.

The attendance rate from towns with peripheral clinics was, on average, $22 \%$ higher than the county average for the relevant speciality. However, periphera clinics took an average of $41 \%$ of the workload for the speciality from the town in which they were sited.

Gas cooking and respiratory disease 留 children: an association. JANE GIBSON, C. DW V. FLOREY, D. G. ALTMAN, AND A. V. SWAG A five-year longitudinal study of primars 
schoolchildren in England and Scotland was started in 1973 in which data on a history of respiratory symptoms and diseases experienced during the past 12 months, the type of fuels used for cooking and heating in the home. and socioeconomic factors were collected in a questionnaire completed annually by the parents. Bronchitis in boys and bronchitis, cough, wheeze and colds going to the chest in girls were more prevalent in children coming from homes where gas was used for cooking than in children coming from homes where electricity was used. The association remained statistically significant when adjustments had been made for the effects of social class and age, and when the interrelationship between diseases and symptoms was considered. Indoor pollution may be the cause of this association.

An epidemiological study of malformations observed at birth in Southern Nigeria. M. M. OGBALU, IAN LECK, AND VALERIE F. HILLIER

Demographic and medical data on 17308 infants (including stillbirths) born in four hospitals in Southern Nigeria during 1970-75 have been abstracted from obstetric unit records, primarily with a view to determining whether neural tube defects and cleft lip are as rare in West Africa itself as in the negroid population of England and the United States, which is of predominantly West African descent. Preliminary analysis suggests that practically all instances of these defects were recorded, and that the proportions of infants affected by them were indeed similar to the figures reported for children of African descent in other continents, thus indicating that the defects' rarity in such children may be genetically determined.

A socio-psychiatric survey among women in a remote Highland community. U. MACLEAN

Psychiatrists have the impression of a high prevalence of depressive illness among Highland women but there has been, to date, no epidemiological survey in the community. In the autumn of 1975 a survey was made of a random sample of 154 women between the ages of 18 and 65 in the small island of North Uist in the Outer Hebrides, using measures developed at the Institute of Psychiatry, London University.

The response rate was good but the results were unexpected. The overall prevalence rates for psychiatric disturb- ance (both cases and borderline cases) are somewhat lower than in London, but the most striking finding has been the relative predominance of anxious rather than depressive conditions. The rate of depression decreases in direct relationship to a measure of 'integration' into the island culture while the rate of anxiety increases.

Doctors, teenagers, and contraception. CHRISTINE FARRELL

The rising number of teenage pregnancies and abortions is currently causing concern. Why do teenagers fail to use contraceptive advice effectively? Although there are a number of possible explanations, ranging from ignorance, fear or deliberate risktaking, this paper concentrates on adolescent attitudes to professional birth control advisers. 1556 teenagers in 12 areas in England and Wales were asked about their attitudes to and their knowledge and use of family planning clinics and general practitioners as providers of birth control information and advice.

In spite of the overall satisfaction expressed by the teenagers with the general practitioner service, there is room for improvement. The characteristics of the girls who had visited only their doctor for advice show that they were generally the older girls ( $76 \%$ were 18 or over) and more likely to be married or engaged $(58 \%$ of married girls, $28 \%$ of engaged girls, and only $13 \%$ of the single girls). It seems clear that older girls who are married, engaged, or having a stable relationship, once they have overcome their initial nervousness, have few reservations about using doctors for birth control advice. The problem, if it can be seen as such, is how to attract single girls and boys into doctors' surgeries, if that is where birth control services for the young should be concentrated.

Social factors and demand for medical care. SHIRLEY A. A. BERESFORD, JANE J. WALLER, MICHAEL H. BANKS, AND CHRISTOPHER J. WALE

The factors which influence an individual's demand for primary medical care include the characteristics of both the individual and of the doctor who is consulted.

A study was designed to investigate personal and social factors associated with demand for care in women aged 20 to 44 years, a group unlikely to suffer from chronic illness. A random sample of women was drawn from the age/sex register of a south London group practice, find information was obtained concerning their daily symptom perception, their anxty level, their social and health characteristiçs, and their consultations for one year.

The social characteristics of the stridy group were described in terms of their association with frequency of demand for medical care. Marital status, social classs, household composition, and fanfly involvement were not associated wigh demand, while absence of basic housing amenities and lack of attachment to neighbourhood were related to $\overrightarrow{\mathrm{ig} h}$ consultation rate.

Explorations of health norms related pregnancy. L. BARIC AND C. MACARTHUkU

A study of pregnant women financed. by the Health Education Council, London, was carried out as a part of a wider stody of socialisation and the transmission of norms concerning health behaviour in general and smoking in particular.

The study examined social noß̧̄ns concerning pregnant women's behaviour (diet, exercise, alcohol consumgtien, medication, and smoking). The studig is a contribution to the methods of meastring norms in terms of their attributes valence, conformity, and coercive power. The findings show that women's behavi depends on three types of norms: gen fal (eating the right foods and alcohol consumption), specific (medication 魚d exertion), and transitional (smokmg) norms. In addition to its contribution-to methodology, the study suggested cerfin implications for health education whichare to turn both general and transitional nofins into specific ones which manifested the highest degree of conformity.

Acute lower respiratory illness in the ifst year of life. $c$. J. WATKINS

There is epidemiological evidence of a permanent reduction of peak expiratery flow rate in infants who have suffered frem bronchitis or pneumonia during the fipst year of life, as reported by their parents at the age of one year. Little informatiofis available about the characteristics of theste illnesses as they appear to the doctor.

The object of this study is to distingựsh clinically those children whose parents report bronchitis or pneumonia during first year of life, from those whose parents report no such history. This is a prospec tive study of infants born to parents istered with two group general practices. 
Details of the clinical characteristics of each respiratory illness, as recorded by the general practitioner, will be related to parentally reported episodes of lower respiratory illness, family history of respiratory and allergic illness, and a number of social variables.

Outpatient consultations and support services. HELEN RUTHVEN

Outpatient consultations in general medicine, general surgery, ear, nose and throat, ophthalmology, and gynaecology in five Scottish Hospitals were reviewed retrospectively from clinical records. Consultations were sampled from all those taking place in the selected specialties and hospitals in 1974. The extent to which a consultation requires laboratory or specialist support services has been assessed and examined in relation to factors such as source of referral and the characteristics of the outpatient episode to which the consultation belongs. The paper examines the feasibility of transferring consultations to a health centre setting.

A prospective survey of the development of coronary heart disease. J. PEMBERTON AND MARY GREIG

The population studied consisted of all the men born in the period 1909-18 on the lists of 10 general practitioners in Belfast. They were examined by clinical, biochemical and electrocardiographic methods in the years 1964-70. 1202 men have bee followed-up for at least five years and 104 have developed angina pectoris or my cardial infarction. The paper presents data showing the relationship between each of 15 continuously distribut variables and 13 categories and the development of coronary heart disease $\mathbf{m}$ the five years after the examination.

Significant associations between the risk of developing coronary heart disease and systolic blood pressure, diastolic blood pressure, tobacco consumption, heigh serum cholesterol, and certain ECE abnormalities were found. 